\author{
Amit KUMAR, Pooja DHIMAN \\ Lovely Professional University, Punjab
}

\title{
RELIABILITY ESTIMATION OF A NETWORK STRUCTURE USING GENERALIZED TRAPEZOIDAL FUZZY NUMBERS
}

\begin{abstract}
Classical sets are used commonly to consider reliability. Because of the uncertainty in the data (which considered in the present paper) classical sets fail to describe the reliability accurately. Uncertainty leads to fluctuation in the actual situation of the structure. Fuzzy logic method attempts to test system reliability with the benefit of membership function. Within this context, specific problems of reasoning-based approaches are studied, explored and correlated with standard reliability approaches. In this paper Generalized Trapezoidal Fuzzy numbers (GTrFN) are used to assess the structure's fuzzy reliability. The reliability of each event is assigned with different level of satisfaction and some improved operations on the generalized trapezoidal fuzzy numbers $(G T r F N)$ are used to calculate the fuzzy boundaries for the resultant reliability of the final event along with the degree of satisfaction. Also the results are compared to demonstrate the application of the improved operations on Generalized Trapezoidal Fuzzy Numbers (GTrFN). The obtained results converge to more precise interval values as compare to the vague fuzzy number.
\end{abstract}

Keywords: classical Sets, fuzzy sets, reliability, Fault Tree Analysis (FTA), Generalized Trapezoidal, fuzzy number, arithmetic operations, DC power supply 


\section{Introduction}

Basically classical set share certain characteristics. The classical set has two groups: members and nonmembers. This is a binary issue. On the other hand, fuzzy set can be considered as an extension and generalization of the basic classical set definition. A fuzzy set have degree of membership between 0 (nonmember) and 1(member). An essential feature is that partial membership is allowed in the fuzzy set that is between 0 and 1. Zadeh [18] proposed fuzzy set theory, which allows the fuzzy boundaries for the replacement of sharp boundaries. The effectiveness of the application of fuzzy sets depends on the user's skill in constructing proper, often extremely accurate membership functions. Chen [2] described the concept of fuzzy sets along with the possibility theory.

The basic elements of reliability are, according to the theory, chance, sufficient performance, time of operation and operating conditions. The reliability of a system is the probability that, under some operating conditions, the system performs a task properly over some time interval. The behavior of any system is thoroughly defined in the sense of probabilities measures. The performance of the top event system is assured and correct as long as the assignment of specific events is based on reliable information. The reliability of the item is the probability that the item executes the task specified [7]. Reliability is a measurement of efficiency in any system. The reliability of the component in a given structure represents the probability of the component's failure. The scope of reliability engineering is extremely broad, encompassing different aspects of engineering technology. The design of any structured framework or structure specifically shows the proper security and functionality of that structure, irrespective of the design theory used. Unfortunately, however, loss is a natural phenomenon associated with the construction of the structure of the object. Several types of redundant components used to increase the stability of the network. We have broadened the use of fault tree analysis to define the most basic factor responsible for structural collapse.

The principle of fault analysis (FTA) has been developed by Bell Telephone Laboratories as a technique used to test the reliability of the Minuteman Launch Control System in 1961. Later, Boeing Corporation modified the concept of application use. Still FTA has been widely used in other fields, such as nuclear power stations, the chemical and aviation industries [4, 8-10]. A fault tree is defined as a model that graphically and logically represents the various combinations of possible events, both faulty and normal, that occur in a structure that leads to unwanted top occurrence. Singer [16] argued that the traditional fault tree would not adhere to tolerances for fault hazard values. Jula et al. [6] explored the application of Boolean to find the reliability of an aircraft electric power system and also improve the fault-tolerance behavior. Furthermore, Furuta and Shiraishi [5] shown that the value of each specific event can be measured through a fuzzy method.

Nevertheless, [17] described the selection of components that contribute as much as possible to the system failure probability is also important in the fault tree research. Mahapatra and Roy [11] used generalized fuzzy numbers in the redundancy allocation to ensure 
the reliability of the series-parallel system. Zhang et al. [19] strengthened the current concept of generalized fuzzy numbers, allowing it a generalization of the concept of fuzzy numbers, and it is noted that many of the results of trapezoidal generalized fuzzy numbers can also be applied to generalized fuzzy numbers. Sharma and Pandey [13] have followed an approach to determining the reliability of the multistate fault tree model by introducing a fuzzy logic. The definition of arithmetic activities on vague sets and vague, set theoretical approach to the study of fault trees, with the assistance of membership and non-membership, was given by Sharma and Pandey [14] on the basis of this methodology. Sharma [15] analyzed the vague reliability of the system. He examined the failure rate using the sugeno's technique for the fuzzy failure rate estimation. Here the vague reliability lies between two heights, which is identical for all components. In the present paper, the different membership functions are associated with each event according to their weightage of participating in the final event. Dhiman and Garg [3] introduced the idea of improved arithmetic operations on generalized fuzzy numbers and conceptual approach to fault tree analysis. With the help of these improved operations, overall device characteristics can be defined. It demonstrates that the reliability of the top event is more precise. And it gives the reliability of the final event at the one membership function, which is more relevant and considerable. The result is shown with the help of graph and the alpha-cuts for the final events are also demonstrated.

The remaining of the article is therefore arranged. In section 2, the notations are defined. In section 3, some preliminaries are defined. In section 4, we discuss briefly the meanings of generalized trapezoidal fuzzy numbers and in section 5, arithmetic operations on general fuzzy numbers and their $\alpha$-cut according to the transformed generalized fuzzy numbers. Section 6 deals with evaluation of reliability of series, parallel and combination of series-parallel networks and section 7 illustrates the proposed methodology to deal with a numerical example of a network system to calculate the final reliability. Section 8 shows results and discussion. At last conclusion is given in the last section 9 and future scope is given in section 10 .

\section{Notations}

Table 1

Notations

\begin{tabular}{|l|l|}
\hline Notation & Description \\
\hline$\widetilde{X}$ & Universal set \\
\hline$\widetilde{A}$ & Fuzzy Set \\
\hline$\mu_{\widetilde{A}}$ & Membership function \\
\hline$\widetilde{A}_{\alpha}$ & Alpha-cuts \\
\hline
\end{tabular}


tab. 1 cont.

\begin{tabular}{|l|l|}
\hline \hline$\widetilde{R}_{S}$ & Reliability of series network \\
\hline$\widetilde{R}_{P}$ & Reliability of parallel network \\
\hline$\widetilde{R}_{C}$ & Reliability of combination of series-parallel network \\
\hline$R_{i}$ & Vague reliability of an event i \\
\hline$\widetilde{R}_{i}$ & Generalized reliability of an event i \\
\hline
\end{tabular}

\section{Preliminaries}

\section{a) Fuzzy Set}

The fuzzy set $\tilde{A}$ in the universe $X$ is interpret as a set of ordered pairs $\left(x, \mu_{\widetilde{A}}\right)$, where $x=$ element of set $\widetilde{A}$ and $\mu_{\widetilde{A}}=$ degree of membership that the element belongs to the set $\widetilde{A}$. Also $\mu_{\widetilde{A}} \in[0,1]$

\section{b) Membership function for the fuzzy set}

Membership defines the fuzziness of the fuzzy set. The membership value is within the range of $[0,1]$. Membership function $\mu$ is a mapping from universal set $X$ to the membership space $M$ and is defined as

$$
\mu: X \rightarrow M
$$

The following are the three key characteristics of the membership category

- Core

In some fuzzy set $\tilde{A}$ the core of the membership function is defined as the universe area, which is characterized by a complete membership in set $\widetilde{A}$. The core of the universe has elements $x$, so that

$$
\mu_{\widetilde{A}}(x)=1
$$

An empty set may form the core of empty set.

\section{- Support}

The membership support function is defined for a fuzzy set A as that region of the universe characterized in set A by a nonzero member. Support is made up of elements $x$ of the universe such that

$$
\mu_{\widetilde{A}}(x)>0
$$




\section{- Boundary}

The boundary of the membership function for the fuzzy set $\mathrm{A}$ is defined as the region of the universe that contains a non-zero but not a complete membership. In other words, the boundary consists of the elements $x$ of the universe, such as

$$
0<\mu_{\widetilde{A}}(x)<1
$$

c) Alpha cuts $(\alpha-$ cuts $)$

Alpha Cut is the most meaningful and widely used concept of fuzzy set theory introduced by Zadeh [18]. When we want to display an element $x$ that normally is set $\widetilde{A}$, we can ask for an element greater than an alpha threshold. This is called alpha-cut.

$$
\begin{gathered}
\widetilde{A}_{\alpha}=\left\{x ; \mu_{A}(x)>\alpha\right\} \quad \text { is called strong alpha cut } \\
\widetilde{A}_{\alpha}=\left\{x ; \mu_{A}(x) \geq \alpha\right\} \text { is called weak alpha cut } \\
\text { Where } 0 \leq \alpha<1
\end{gathered}
$$

\section{d) Convex Fuzzy Set}

A fuzzy set $\widetilde{A}=\left\{\left(x, \mu_{\widetilde{A}}\right), x \in X\right\}$ which satisfies the trailing inequality

$$
\mu_{\widetilde{A}}\left(\lambda x_{1}+(1-\lambda) x_{2}\right) \geq \min \left(\mu_{\widetilde{A}}\left(x_{1}\right), \mu_{\widetilde{A}}\left(x_{2}\right)\right) ; x_{1}, x_{2} \in X \text { and } \lambda \in[0,1]
$$

is known as convex fuzzy set. If this inequality does not hold, the fuzzy set is known as non-convex fuzzy set.

\section{e) Normal Fuzzy Set}

A fuzzy set is normal set when there is at least one element in the universal set $X$ so that its membership function is one.

\section{f) Fuzzy Number (FN)}

A convex, normal (if there is at least one number on Real line such that its membership function is unity) membership function on a Real line is called fuzzy number [20] The respective membership function $\mu$ for a fuzzy number $\widetilde{A}=\left(a_{1}, a_{2}, a_{3}\right)$ is given as

$$
\mu_{\widetilde{A}}(x)=\left\{\begin{array}{l}
f_{1}(x) ; x \in\left(a_{1}, a_{2}\right) \\
1 ; x \in a_{2} \\
f_{2}(x) ; x \in\left(a_{2}, a_{3}\right) \\
0 ; \text { otherwise }
\end{array}\right.
$$


$f_{1}(x), f_{2}(x)$ are monotonic, continuous from the left and right.

g) Trapezoidal Fuzzy Number (TrFN)

A trapezoidal fuzzy number is a fuzzy number $\widetilde{A}=(a, b, c, d)$ if its membership function $\mu_{\widetilde{A}}$ is given by

$$
\mu_{\widetilde{A}}(x)=\left\{\begin{array}{l}
\left(\frac{x-a}{b-a}\right) ; \text { if } a \leq x \leq b \\
1 ; \text { ifb } \leq x \leq c \\
\left(\frac{d-x}{d-c}\right) ; \text { ifc } \leq x \leq d \\
0 ; \text { otherwise }
\end{array}\right.
$$

The alpha-cut is given by the closed interval

$$
\widetilde{A}_{\alpha}=\left[\widetilde{A}_{\alpha}^{L}, \widetilde{A}_{\alpha}^{R}\right]=[a+\alpha(b-a), d-\alpha(d-c)]
$$

\section{Generalized Trapezoidal Fuzzy Numbers (GTrFN)}

A fuzzy numbers $\widetilde{A}=\langle(a, b, c, d ; \omega) \mid a, b, c, d \in R\rangle$, if the corresponding membership function $\mu_{\widetilde{A}}(x): R \rightarrow[0,1]$ satisfies the following conditions, is said to be generalized trapezoidal fuzzy number (GTrFN).

It is piecewise continuous.

For all $x \in(-\infty, a] \cup[d, \infty)$, it is zero.

It is rising strictly on $[a, b]$ and declines sharply on $[b, c]$.

For all $x \in[b, c], \mu_{\widetilde{A}}(x)=\omega$ where $0<\omega \leq 1$.

The membership function $\mu_{A}(x): R \rightarrow[0,1]$ is defined as

$$
\mu_{\tilde{A}}(x)=\left\{\begin{array}{l}
\omega\left(\frac{x-a}{b-a}\right) ; \text { if } a \leq x \leq b \\
\omega ; \text { ifb } \leq x \leq c \\
\omega\left(\frac{d-x}{d-c}\right) ; \text { if } c \leq x \leq d \\
0 ; \text { otherwise }
\end{array}\right.
$$


The alpha cut is given by

$$
\widetilde{A}_{\alpha}=\left[\widetilde{A}_{\alpha}^{L}, \widetilde{A}_{\alpha}^{R}\right]=\left[a+\frac{\alpha}{\omega}(b-a), c-\frac{\alpha}{\omega}(c-b)\right], \alpha \in(0, \omega]
$$

\section{Improved Arithmetic Operations between Generalized Trapezoidal Fuzzy Numbers}

Let $\mathrm{A}$ and $\mathrm{B}$ be two generalized, fuzzy trapezoidal numbers with membership functions $\mu_{\widetilde{A}}$ and $\mu_{\widetilde{B}}$.

Assume $\widetilde{A}=\left(a_{1}, b_{1}, c_{1}, d_{1} ; \omega_{1}\right)$ and $\widetilde{B}=\left(a_{2}, b_{2}, c_{2}, d_{2} ; \omega_{2}\right), \quad 0 \leq \omega_{1}, \omega_{2} \leq 1$ such that $\omega_{1} \leq \omega_{2}$.

What one should write as

$$
\begin{aligned}
& \mu_{\widetilde{A}}(x)=\left\{\begin{array}{l}
\omega_{1}\left(\frac{x-a_{1}}{b_{1}-a_{1}}\right) ; i f a_{1} \leq x \leq b_{1} \\
\omega_{1} ; i f b_{1} \leq x \leq c_{1} \\
\omega_{1}\left(\frac{d_{1}-x}{d_{1}-c_{1}}\right) ; i f c_{1} \leq x \leq d_{1} \\
0 ; \text { otherwise }
\end{array} ; \mu_{\widetilde{B}}(x)=\left\{\begin{array}{l}
\omega_{2}\left(\frac{x-a_{2}}{b_{2}-a_{2}}\right) ; i f a_{2} \leq x \leq b_{2} \\
\omega_{2} ; i f b_{2} \leq x \leq c_{2} \\
\omega_{2}\left(\frac{d_{2}-x}{d_{2}-c_{2}}\right) ; i f c_{2} \leq x \leq d_{2} \\
0 ; \text { otherwise }
\end{array}\right.\right. \\
& \omega=\min \left(\omega_{1}, \omega_{2}\right)
\end{aligned}
$$

Then make a $\omega$ cut of fuzzy number to turn $\widetilde{B}=\left(a_{2}, b_{2}, c_{2}, d_{2} ; \omega_{2}\right)$ into a generalized fuzzy number $\widetilde{B}^{*}=\left(a_{2}, b_{2}^{*}, c_{2}^{*}, d_{2} ; \omega\right)$ as shown in fig. 1 , that can be defined as

$$
\begin{gathered}
\widetilde{B}^{*}=\left(a_{2}, b_{2}^{*}, c_{2}^{*}, d_{2} ; \omega\right), \quad b_{2}^{*}=a_{2}+\frac{\omega}{\omega_{2}}\left(b_{2}-a_{2}\right) \\
\text { and } c_{2}^{*}=d_{2}-\frac{\omega}{\omega_{2}}\left(d_{2}-c_{2}\right)
\end{gathered}
$$

Now the alpha-cut corresponding to $\widetilde{A}$ and $\widetilde{B}$ is given by

$$
\begin{aligned}
& \widetilde{A}_{\alpha}=\left[\widetilde{A}_{\alpha}^{L}, \widetilde{A}_{\alpha}^{R}\right]=\left[a_{1}+\frac{\alpha}{\omega_{1}}\left(b_{1}-a_{1}\right), d_{1}-\frac{\alpha}{\omega_{1}}\left(d_{1}-c_{1}\right)\right], \alpha \in\left(0, \omega_{1}\right], \omega_{1} \in[0,1] \\
& \widetilde{B}_{\alpha}^{*}=\left[\widetilde{B}_{\alpha}^{* L}, \widetilde{B}_{\alpha}^{* R}\right]=\left[a_{2}+\frac{\alpha}{\omega_{1}}\left(b_{2}^{*}-a_{2}\right), d_{2}-\frac{\alpha}{\omega_{1}}\left(d_{2}-c_{2}^{*}\right)\right], \alpha \in(0, \omega], \omega \in[0,1]
\end{aligned}
$$



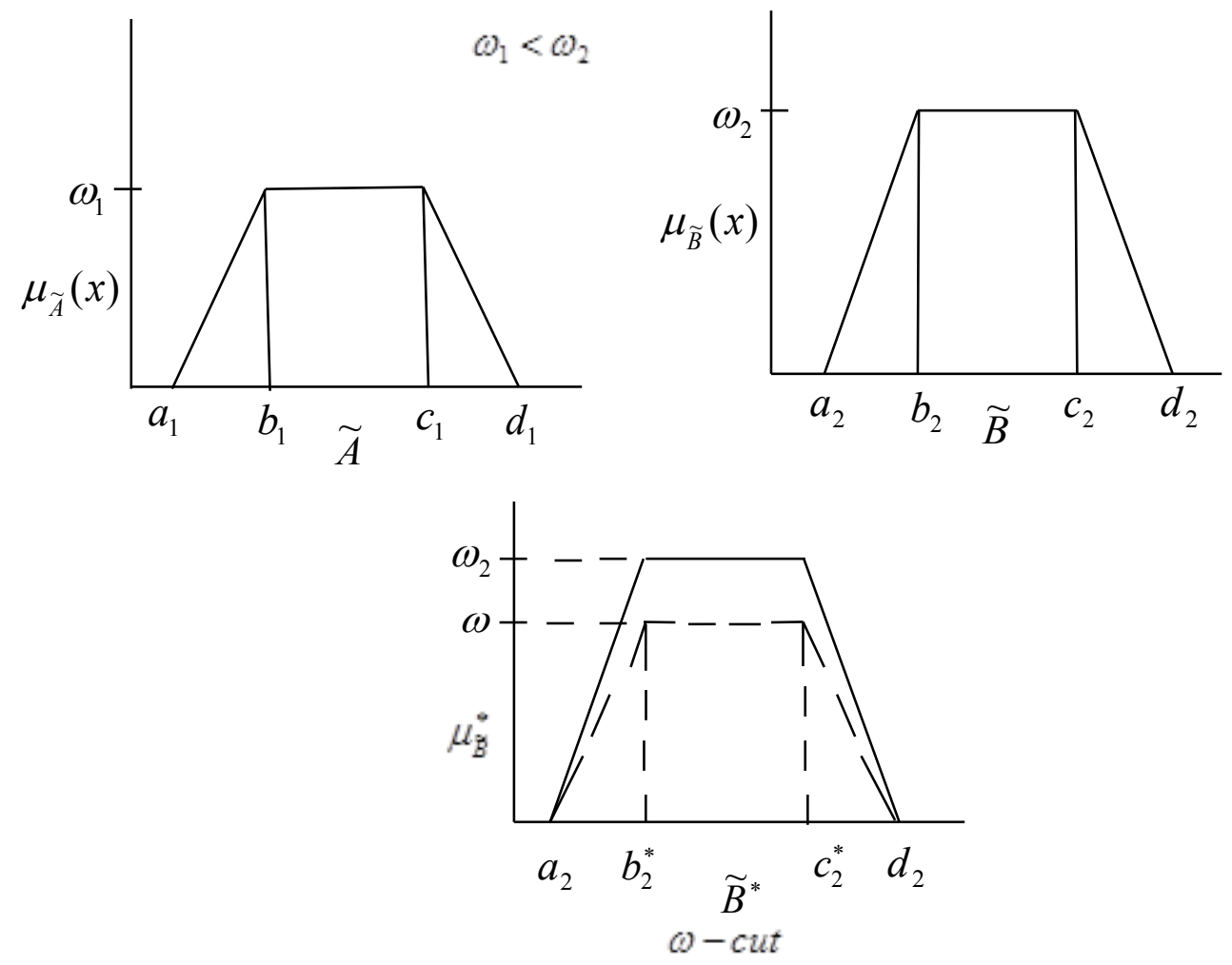

Fig. 1. Transforming $\widetilde{B}_{\text {to }} \widetilde{B}^{*}$

- Improved Arithmetic operations are given as

a) Addition of two generalized fuzzy numbers

$$
\begin{aligned}
& \widetilde{A}+\widetilde{B}=\left(a_{1}+a_{2}, b_{1}+a_{2}+\omega\left(\frac{b_{2}-a_{2}}{\omega_{2}}\right), c_{1}+d_{2}-\omega\left(\frac{d_{2}-c_{2}}{\omega_{2}}\right), d_{1}+d_{2}\right) \\
& \text { or } \\
& \widetilde{A}+\widetilde{B}^{*}=\left(a_{1}+a_{2}, b_{1}+b_{2}^{*}, c_{1}+c_{2}^{*}, d_{1}+d_{2}\right)
\end{aligned}
$$


b) Subtraction of two generalized fuzzy numbers

$$
\begin{aligned}
& \widetilde{A}+(-\widetilde{B})=\left(a_{1}, b_{1}, c_{1}, d_{1}\right)+\left(-d_{2},-c_{2},-b_{2}, a_{2}\right) \\
& \widetilde{A}-\widetilde{B}=\left(a_{1}-d_{2}, b_{1}-d_{2}+\omega\left(\frac{d_{2}-c_{2}}{\omega_{2}}\right), c_{1}-a_{2}-\omega\left(\frac{b_{2}-a_{2}}{\omega_{2}}\right), d_{1}-a_{2}\right)
\end{aligned}
$$

or

$\widetilde{A}-\widetilde{B}^{*}=\left(a_{1}-d_{2}, b_{1}-c_{2}^{*}, c_{1}-b_{2}^{*}, d_{1}-a_{2}\right)$

c) Multiplication of two generalized fuzzy numbers

$$
\widetilde{A} \cdot \widetilde{B}=\left(a_{1} \cdot a_{2}, b_{1} \cdot a_{2}+\omega\left(\frac{b_{1} \cdot b_{2}-b_{1} \cdot a_{2}}{\omega_{2}}\right), c_{1} \cdot d_{2}-\omega\left(\frac{c_{1} \cdot d_{2}-c_{1} \cdot c_{2}}{\omega_{2}}\right), d_{1} \cdot d_{2}\right)
$$

or

$\widetilde{A} \cdot \widetilde{B}^{*}=\left(a_{1} \cdot a_{2}, b_{1} \cdot b_{2}^{*}, c_{1} \cdot c_{2}^{*}, d_{1} \cdot d_{2}\right)$

d) Division of two generalized fuzzy numbers

$$
\begin{aligned}
& \widetilde{A} / \widetilde{B}=\widetilde{A} \times(1 / \widetilde{B})=\left(a_{1}, b_{1}, c_{1}, d_{1}\right) \times\left(1 / d_{2}, 1 / c_{2}, 1 / b_{2}, 1 / a_{2}\right) \\
& \widetilde{A} / \widetilde{B}=\left(a_{1} / d_{2}, b_{1} / d_{2}+\omega\left(\frac{b_{1} / c_{2}-b_{1} / d_{2}}{\omega_{2}}\right), c_{1} / a_{2}+\omega\left(\frac{c_{1} / b_{2}-c_{1} / a_{2}}{\omega_{2}}\right), d_{1} / a_{2}\right)
\end{aligned}
$$

or

$\widetilde{A} / \widetilde{B}^{*}=\left(a_{1} / d_{2}, b_{1} / c_{2}^{*}, c_{1} / b_{2}^{*}, d_{1} / a_{2}\right)$

\section{Evaluation of Reliability of Series, Parallel and Combination of Series-Parallel Networks}

- Series Networks:-This arrangement is a structure where components form a serial network [1]. If any of the components fails, the series structure fails ultimately. If n-components $R_{1}, R_{2}, R_{3} \ldots R_{n}$ are arranged in series as shown in fig. 2. 


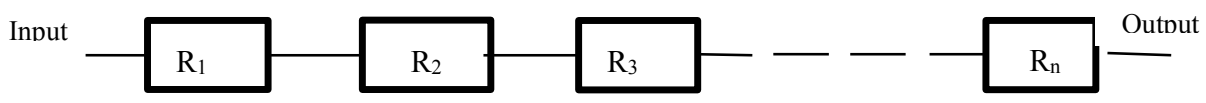

Fig. 2. Series Network

The reliability of such kind of arrangement is given as:

$$
\widetilde{R}_{S}=\widetilde{R}_{1} \otimes \widetilde{R}_{2} \otimes \widetilde{R}_{3} \otimes \ldots \otimes \widetilde{R}_{n}
$$

- Parallel Networks:-If n-components i.e. $R_{1}, R_{2}, R_{3} \ldots R_{n}$ are arranged in parallel settlement. When each part fails, the entire structure fails.

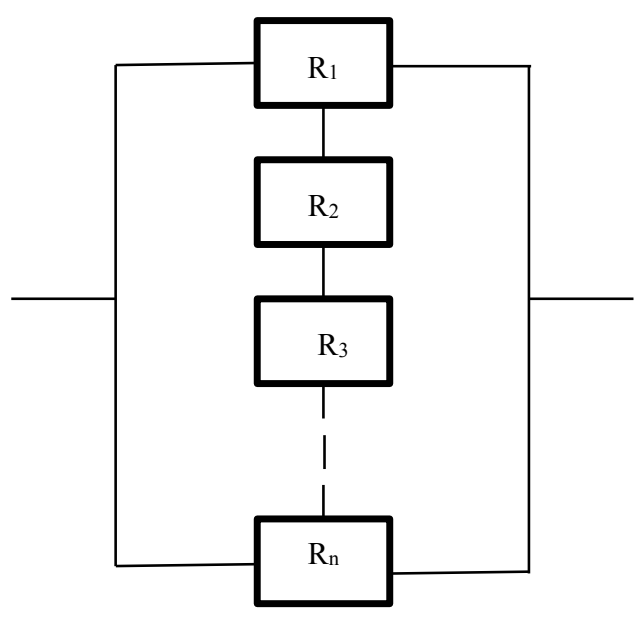

Fig. 3. Parallel Network

$$
\widetilde{R}_{P}=1-\left(1-\widetilde{R}_{1}\right)\left(1-\widetilde{R}_{2}\right)\left(1-\widetilde{R}_{3}\right) \ldots\left(1-\widetilde{R}_{n}\right)
$$

- Combination of series-parallel network: In the next figure, a series-parallel network including ' $n$ ' units in parallel arrangement along with each network contains ' $\mathrm{m}$ ' subunits. The reliability of such type of the arrangement network structure is given by $\widetilde{R}_{C}$ is calculated as 


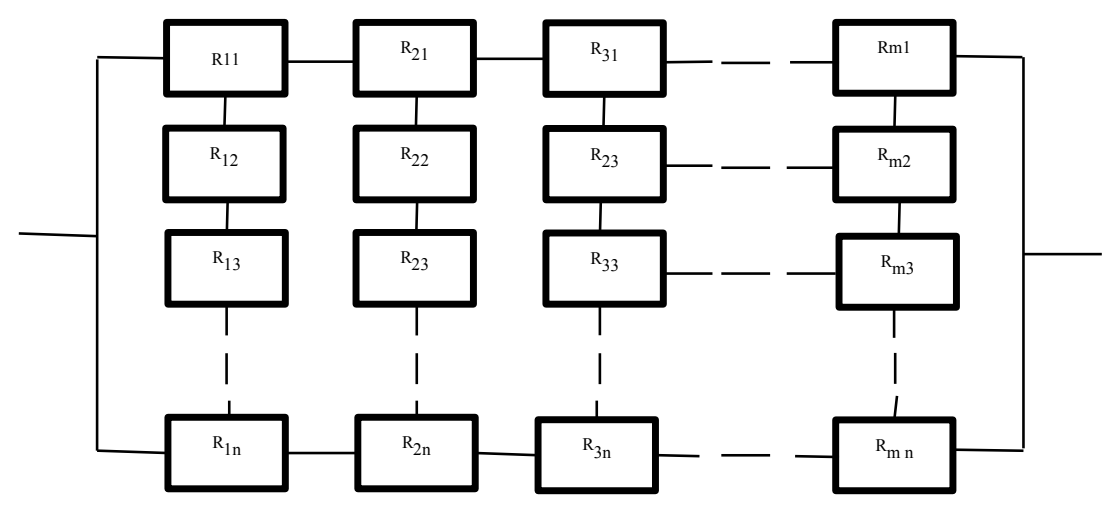

Fig. 4. Series-Parallel Network

$$
\begin{gathered}
R_{C}={ }_{j=1}^{n} \otimes\left(1-{ }_{i=1}^{m}\left(1-\widetilde{R}_{i j}\right)\right) \\
\widetilde{R}_{C}=\left(1-\left(1-\widetilde{R}_{11}\right)\left(1-\widetilde{R}_{12}\right)\left(1-\widetilde{R}_{13}\right) \ldots\left(1-\widetilde{R}_{1 n}\right)\right) \otimes\left(1-\left(1-\widetilde{R}_{21}\right)\left(1-\widetilde{R}_{22}\right)\left(1-\widetilde{R}_{23}\right) \ldots\left(1-\widetilde{R}_{2 n}\right)\right) \\
\otimes\left(1-\left(1-\widetilde{R}_{31}\right)\left(1-\widetilde{R}_{32}\right)\left(1-\widetilde{R}_{33}\right) \ldots\left(1-\widetilde{R}_{3 n}\right)\right) \ldots \otimes\left(1-\left(1-\widetilde{R}_{m 1}\right)\left(1-\widetilde{R}_{m 2}\right)\left(1-\widetilde{R}_{m 3}\right) \ldots\left(1-\widetilde{R}_{m n}\right)\right)
\end{gathered}
$$

\section{Numerical Computations}

A strong current special machine needs a continuous DC power supply for a particular period of time to perform its required function. Model from [14] has been taken to illustrate the methodology. In this structure we will calculate the reliability for the event such that there is no DC supply to the machine and this event happens due to the failure of some certain events. All the events participate to carry out the resultant top event. The failure tree shown in fig. 4 is a representation of all possible incidents, their logical configurations and their relation to failure of the structure. The lowest level events are called basic incidents. The combinations of basic events such as events are described as intermediate events. The reliabilities of failure of basic events are combined to obtain the reliability of failure of intermediate events and the failure of the structure.

Events participating in the failure of the structure are given as

$E_{1=}$ Grid Failure

$E_{2}=$ Substation Failure

$E_{3}=$ Switch of the DC supply

$E_{4}=$ Convertor 1 fails

$E_{5}=$ Convertor 2 fails 
$E_{6}=$ Power supply to the convertor

$E_{7}=$ Both convertor fails

$E_{8}=$ No DC supply to the machine

$\widetilde{R}_{i}=$ Reliability of each event $E_{i}$

No DC Supply to the machine

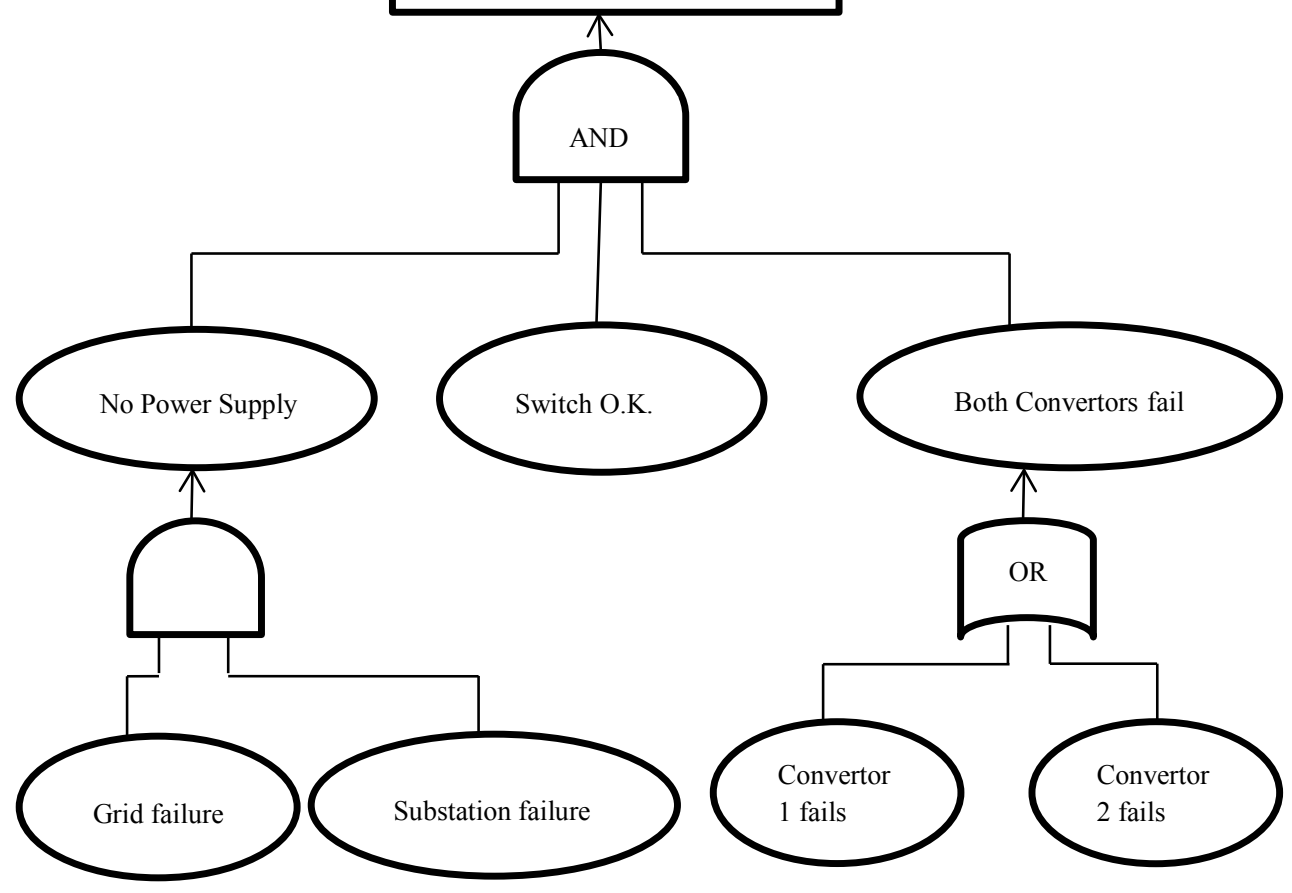

Fig. 5. Fault Tree for DC Supply to the machine

Let the vague reliability of the events are defined with membership and non-membership functions with vague boundaries.

$$
\begin{aligned}
& R_{i}=\left(\mu ; \omega_{1}\right),\left(v ; \omega_{2}\right) \\
& R_{1}=(0.6931,0.7169,0,0.7989,0.8289 ; 0.7),(0.5898,0.6787,0.7804,0.8995 ; 0.8) \\
& R_{2}=(0.6735,0.7865,0,0.8245,0.8925 ; 0.7),(0.6145,0.6736,0.7815,0.9125 ; 0.8) \\
& R_{3}=(0.7818,0.8025,0,0.8992,0.9169 ; 0.7),(0.6735,0.8052,0.8289,0.9136 ; 0.8) \\
& R_{4}=(0.7236,0.8034,0,0.8129,0.8912 ; 0.7),(0.6712,0.7028,0.8125,0.9024 ; 0.8) \\
& R_{5}=(0.6134,0.6329,0,0.71848,0.8127 ; 0.7),(0.6022,0.7125,0.8043,0.9134 ; 0.8)
\end{aligned}
$$


Representation of each vague reliability $\widetilde{R}_{i}$ with membership and non-membership functions

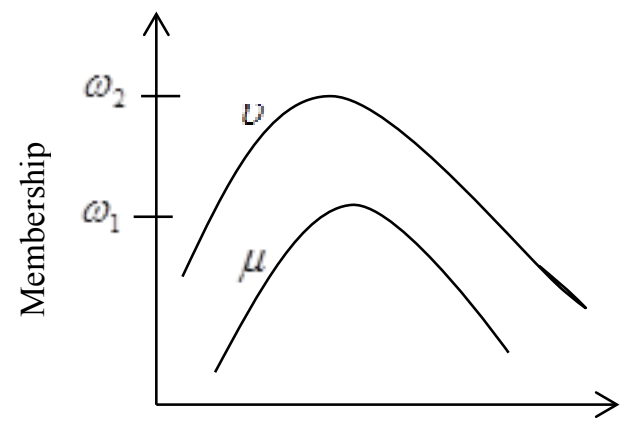

Reliability

Fig. 6. Vague membership function of Reliability of each unit

First of all given data is converted to the generalized trapezoidal number by taking the average of lower and upper value. Also different level of satisfaction is assigned with every generalized fuzzy number. Level of satisfaction is assigned according to the participation of each event for the resultant of top event.

$$
\begin{aligned}
& \widetilde{R}_{1}=\left(\frac{0.6931+0.5898}{2}, \frac{0.7169+0.6787}{2}, \frac{0.7989+0.7804}{2}, \frac{0.8289+0.8995}{2} ; 0.70\right) \\
& \widetilde{R}_{2}=\left(\frac{0.6735+0.6145}{2}, \frac{0.7865+0.6736}{2}, \frac{0.8245+0.7815}{2}, \frac{0.8925+0.9125}{2} ; 0.75\right) \\
& \widetilde{R}_{3}=\left(\frac{0.7818+0.6735}{2}, \frac{0.8025+0.8052}{2}, \frac{0.8992+0.8289}{2}, \frac{0.9169+0.9136}{2} ; 0.80\right) \\
& \widetilde{R}_{4}=\left(\frac{0.7236+0.6712}{2}, \frac{0.8034+0.7028}{2}, \frac{0.8129+0.8125}{2}, \frac{0.8912+0.9024}{2} ; 0.75\right) \\
& \widetilde{R}_{5}=\left(\frac{0.6134+0.6022}{2}, \frac{0.6329+0.7125}{2}, \frac{0.71848+0.8043}{2}, \frac{0.8127+0.9134}{2} ; 0.70\right)
\end{aligned}
$$




$$
\begin{aligned}
& \widetilde{R}_{1}=(0.64145,0.6978,0,0.78965,0.8642 ; 0.7) \\
& \widetilde{R}_{2}=(0.644,0.73005,0,0.803,0.9025 ; 0.75) \\
& \widetilde{R}_{3}=(0.72765,0.80385,0,0.86405,0.91525 ; 0.8) \\
& \widetilde{R}_{4}=(0.6974,0.7531,0,0.8127,0.8968 ; 0.75) \\
& \widetilde{R}_{5}=(0.6078,0.6727,0,0.76139,0.86305 ; 0.7)
\end{aligned}
$$

Representation of each reliability $\widetilde{R}_{i}$ in generalized trapezoidal fuzzy number with membership function $\mu$ and degree $\omega_{i}$

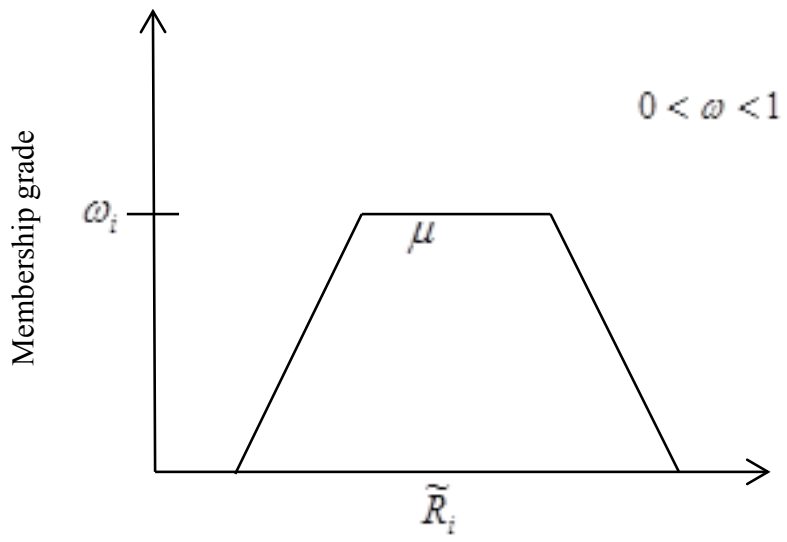

Reliability

Fig. 7. Generalized Trapezoidal Fuzzy Number of Reliability of each unit

Generalized Fuzzy number by making $\omega-c u t$ for all the numbers by taking $\omega=\omega_{\min }\left(\omega_{i}{ }^{\prime} s\right), i=1,2,3,4,5$

$$
\begin{aligned}
\omega=\omega_{\min }(0.7,0.75,0.8,0.75,0.7)=0.7 \\
\widetilde{R}_{1}=(0.64145,0.6978,0,0.78965,0.8642 ; 0.7) \\
\widetilde{R}_{2}^{*}=(0.644,0.724313,0,0.809633,0.9025 ; 0.7) \\
\widetilde{R}_{3}^{*}=(0.72765,0.794325,0,0.87045,0.91525 ; 0.7) \\
\widetilde{R}_{4}^{*}=(0.6974,0.749387,0,0.818307,0.8968 ; 0.7) \\
\widetilde{R}_{5}=(0.6078,0.6727,0,0.76139,0.86305 ; 0.7)
\end{aligned}
$$




$$
\begin{gathered}
\widetilde{R}_{6}=\widetilde{R}_{1} \otimes \widetilde{R}_{2} \\
\widetilde{R}_{7}=1-\left(1-\widetilde{R}_{4}\right)\left(1-\widetilde{R}_{5}\right) \\
\widetilde{R}_{8}=\widetilde{R}_{6} \otimes \widetilde{R}_{7} \otimes \widetilde{R}_{8} \\
\widetilde{R}_{6}=(0.4131,0.5054,0.6393,0.7799) \\
\widetilde{R}_{7}=(0.8813,0.9180,0.9566,0.9859) \\
\widetilde{R}_{8}=(0.2649,0.3685,0.5323,0.7037)
\end{gathered}
$$

\section{Results and discussion}

The graph of the reliability of the network structure is given in fig. 8 and also the alpha cuts are shown in tab. 2, which clearly defines the degree of satisfaction (alpha) for every interval value of reliability.

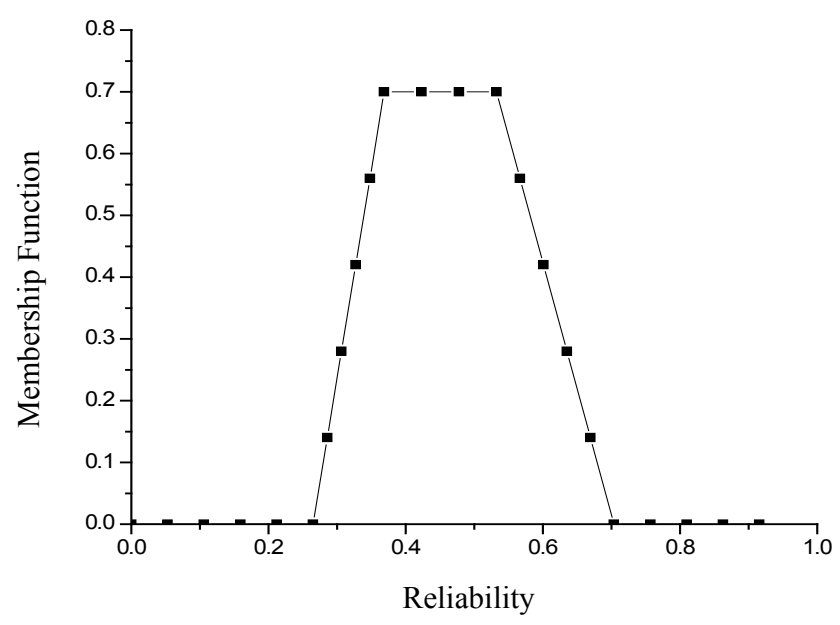

Fig. 8. Reliability of the Network Structure with $70 \%$ degree satisfaction 
Table 2

\section{Alpha cuts of reliability}

\begin{tabular}{|c|c|c|}
\hline Alpha $(\alpha)$ & Left $\alpha$-cut, $\widetilde{R}_{\alpha}^{L}$ & Right $\alpha$-cut, $\widetilde{R}_{\alpha}^{R}$ \\
\hline 0.7 & 0.3685 & 0.5323 \\
\hline 0.6 & 0.3537 & 0.5568 \\
\hline 0.5 & 0.3389 & 0.5813 \\
\hline 0.4 & 0.3241 & 0.6058 \\
\hline 0.3 & 0.3093 & 0.6302 \\
\hline 0.2 & 0.2945 & 0.6547 \\
\hline 0.1 & 0.2797 & 0.6792 \\
\hline 0 & 0.2649 & 0.7037 \\
\hline
\end{tabular}

\section{Conclusions}

In this paper, a structure with the arrangement of combination of series-parallel network is taken into account to illustrate the application of Generalized Trapezoidal fuzzy Numbers (GTrFN) over the Trapezoidal vague fuzzy numbers (TrapVaFN). In this paper, the reliability of the network structure is calculated and shown in fig. 8 and alpha cut is given in tab. 1. It can be observed from the fig. 5 that the reliability for the final event lies in the interval $(0.3685,0.5323)$ with the highest membership grade 0.7 . It is clear that the degree of satisfaction for the reliability $(0.3685,0.5323)$ of top event is $70 \%$. This describes the unreliability of the system such that no current supply to the machine and it is less as compare to the result of [14]. Clearly, it shows that generalized trapezoidal fuzzy number and their improved arithmetic operations gives the more precise and improved result as compare to the vague boundaries. Here authors have been taken one membership grade for the reliability of final/top event which is more convenient for the further realistic decision.

\section{Future Scope of the work}

This work can be extended to generalized parabolic numbers to obtain the reliability with non-linear parabolic fuzzy number and weighted generalized parabolic numbers. 


\section{References}

1. Balagurusamy E.: Reliability Engineering. Tata McGraw-Hill Education Private Limited, 1984.

2. Cheng C.H., Mon D.L.: Fuzzy system reliability analysis by possibility. Microelectron Reliability, 33:587597. 1993.

3. Dhiman P., Garg H.: Reliability analysis of an industrial system using improved arithmetic operations. M.Sc. thesis, Thapar University, 2016.

4. Eisenack K., Kropp J.: Assessment of management options in marine fisheries by qualitative modeling techniques. Mar Pollut Bull 43:215-224, 1984.

5. Furuta H., Shiraishi N.: Fuzzy importance in fault tree analysis. Fuzzy Sets System 12:205-213, 1984.

6. Jula N., Cepisca C., Covrig M., Racuciu C.: Boolean applications in aircraft electric power systems analysis. 2nd European Computing Conference(ECC'08), Malta, 2008.

7. Kales P.: Reliability: for technology, engineering, and management. Prentice-Hall, Englewood Cliffs, 1998.

8. Lee C., Lu T.C., Lee N.P., Chung U.K.: The study of strategy on new equipment maintenance. Fuzzy Sets Math, 13:37-44, 1999.

9. Liang G.S., Wang M.J.J.: Fuzzy fault tree analysis using failure possibility. Microelectron Reliability 33:587-597, 1993.

10. Lin C.T., Wang M.J.: Hybrid fault tree analysis using fuzzy sets. Reliability Engineering System Safety, 58:205-213, 1997.

11. Mahapatra G.S., Roy T.K.: Optimal Redundancy Allocation in Series-Parallel System using Generalized Fuzzy Number, 27(1):1-20, 2011.

12. Mon D.L., Cheng C.H.: Fuzzy system reliability analysis by interval of confidence. Fuzzy Sets System, 56:29-35, 1993.

13. Sharma M.K., Pandey D.: Reliability analysis of multistate fault tree model. Mathematics Today, 25:7-21, 2009.

14. Sharma M.K., Pandey D.: Vague Set Theoretic Approach to Fault Tree Analysis. Journal of International Academy of Physical Sciences, 14(1):1-14, 2010.

15. Sharma M.K.: Vague Reliability of a Network System Using Sugeno's Fuzzy Failure Rates. IOSR Journal of Engineering (IOSRJEN) 8(12):38-48, 2018.

16. Singer D.: A fuzzy set approach to fault tree and reliability analysis. Fuzzy Sets System, 34:145-155, 1990.

17. Suresh P.V., Babar A.K., Raj V.V.: Uncertainty in fault tree analysis: a fuzzy approach. Fuzzy Sets Systems, 83:135-141, 1996.

18. Zadeh L.A.: Fuzzy Sets. Information and Control, 8(3):338-353, 1965.

19. Zhang D.L., Guo C., Chen D.: On generalized fuzzy numbers. Iranian Journal of Fuzzy Sets, 16(1):61-73, 2019.

20. Zimmermann H.: Fuzzy Set Theory and its applications. Kluwer Academic Publishers, 2013. 